\title{
Erratum zu ,Time to find out what Brexit really means“
}

\section{Anand Menon • Matthew Bevington}

Online publiziert: 1 . Juni 2021

(C) Der/die Autor(en) 2021

\section{Erratum zu:}

\section{Z Außen Sicherheitspolit 2021}

https://doi.org/10.1007/s12399-021-00844-x

Der Artikel „Time to find out what Brexit really means“ von Anand Menon und Matthew Bevington wurde ursprünglich am 26. April 2021 ohne „Open Access“ online auf der Internetplattform des Verlags publiziert. Die Autoren haben sich jedoch nachträglich für eine „Open Access“-Veröffentlichung entschieden. Das Urheberrecht des Artikels wurde deshalb 13. Mai 2021 in (C) The Author(s) 2021 geändert.

Die Online-Version des Originalartikels ist unter https://doi.org/10.1007/s12399-021-00844-x zu finden.

Anand Menon $(\bowtie)$

UK in a Changing Europe, 22 Kingsway, London, WC2B 6LE, Großbritannien

E-Mail: anand.menon@ukandeu.ac.uk

Matthew Bevington

Global Counsel, 5 Welbeck Street, London, W1G 9YQ, Großbritannien

E-Mail: m.bevington@global-counsel.com 